\title{
Artificial Neural Networks Model for Predicting Ultimate Analysis using Proximate Analysis of Coal
}

\author{
J. Krishnaiah \\ Research and Development \\ Bharat Heavy Electricals Ltd. \\ Tiruchirapalli
}

\author{
A. Lawrence \\ Central Plant Lab \\ Bharat Heavy Electricals Ltd. \\ Tiruchirapalli
}

\author{
R. Dhanuskodi \\ Research and Development \\ Bharat Heavy Electricals Ltd. \\ Tiruchirapalli
}

\begin{abstract}
In the fossil fuel (coal) based power plants, for estimating the combustion air requirement and for ensuring effective combustion of coal, it is very essential to know the elemental composition of the coal that is fired. Ultimate analysis is the process to be performed to know elemental composition of the coal collected. The ultimate analysis is costly, time-taking and also cumbersome in nature and therefore at the power-plants only gross-level coal compositions are estimated which is called proximate analysis. Based on the gross-level compositions of the coal, the elemental compositions are estimated using standard empirical formulae. The relationship between the gross level composition (i.e. proximate analysis) and the elemental level composition (i.e. ultimate analysis) is nonlinear, whereas the empirical formulae are linear assumptions which may lead to erroneous estimations. The empirical formulae based erroneous estimations lead to variation in the combustion behavior and thereby leading to suboptimal performance of the boilers. To achieve better control on the boilers and thereby to achieve better performance, accurate computation of elemental composition is required. In this article, we suggest a method to compute ultimate analysis based on the proximate analysis information using Artificial Neural Network model (ANN). The predictions of ANN and empirical models have been compared. It is found that the ANN prediction is in very good agreement with lab data than the predictions of empirical model.
\end{abstract}

\section{General Terms}

Predicting Coal properties using Artificial Neural Networks Model

\section{Keywords}

Artificial Neural Networks, Proximate Analysis, Ultimate Analysis of coal, Combustion

\section{INTRODUCTION}

India is the third largest producer and consumer of coal in the world. Coal finds wide usage in many industries as main fuel for the process. Thermal plants are the major users of coal. Coal is an extremely complex material and exhibits a wide range of physical property and chemical properties. The rapidly expanding use of variety of coal at present made it necessary to devise acceptable method for coal analysis. As part of the coal evaluation, new methods/correlations are continuously developed to increase the accuracy, reduce the time of analysis and cost. Ultimate analysis, one of the important coal characterization data represents the elemental composition of organic material in coal in terms of CARBON (C), NITROGEN (N), HYDROGEN (H), SULPHUR(S) and OXYGEN (O). The information about the elemental composition of coal is very important from the design, operational and environmental points of view. Considering the investment and time involved, many coal users may not be having the equipment to carry out the ultimate analysis which estimates the elemental composition. However, the proximate analysis is cheaper and easily available in any plant. But to estimate or compute various controlling parameters in the boilers, the ultimate analysis is an important information. To overcome this limitation many empirical formulae have been devised. The relationships between the gross level composition (i.e. proximate analysis) and the elemental level composition (i.e. ultimate analysis) is nonlinear, whereas the available empirical formulae are based on linear assumptions which may lead to erroneous estimations. Hence the empirical formulae based erroneous estimations lead to variation in the combustion behavior and thereby leading to suboptimal performance of the boilers. To achieve better control on the boilers and thereby to achieve better performance, accurate computation of elemental composition is required. In this article, we suggest a method to compute ultimate analysis based on the proximate analysis information using Artificial Neural Network model (ANN). Around 150 lab analysis datapoints on coal for which both ultimate and proximate information is available had been used to train and test the ANN model.

Composition of proximate analysis is represented by $\%$ Ash, \%Fixed Carbon, \%Moisture and \% Volatile Material. Based on the collected dataset, the average distribution of these components in the coal is shown in Figure 1(a). Whereas, Ultimate analysis is represented by the elemental analysis containing \%Carbon, \%Hydrogen, \%Nitrogen, \%Oxygen and $\%$ Sulphur [1]. The average distribution of these elements in the coal for the collected dataset is shown in Figure 1(b). Different approaches to proximate analysis by thermogravimetry analysis on various coals are discussed and comparisons of these results with ASTM analysis can be found in [2]. In the following sections, the model architecture of the developed ANN model and the results obtained are discussed briefly. Basic need of the proposed model is to predict the elemental composition of ultimate analysis based on the given overall composition information of the proximate analysis. 

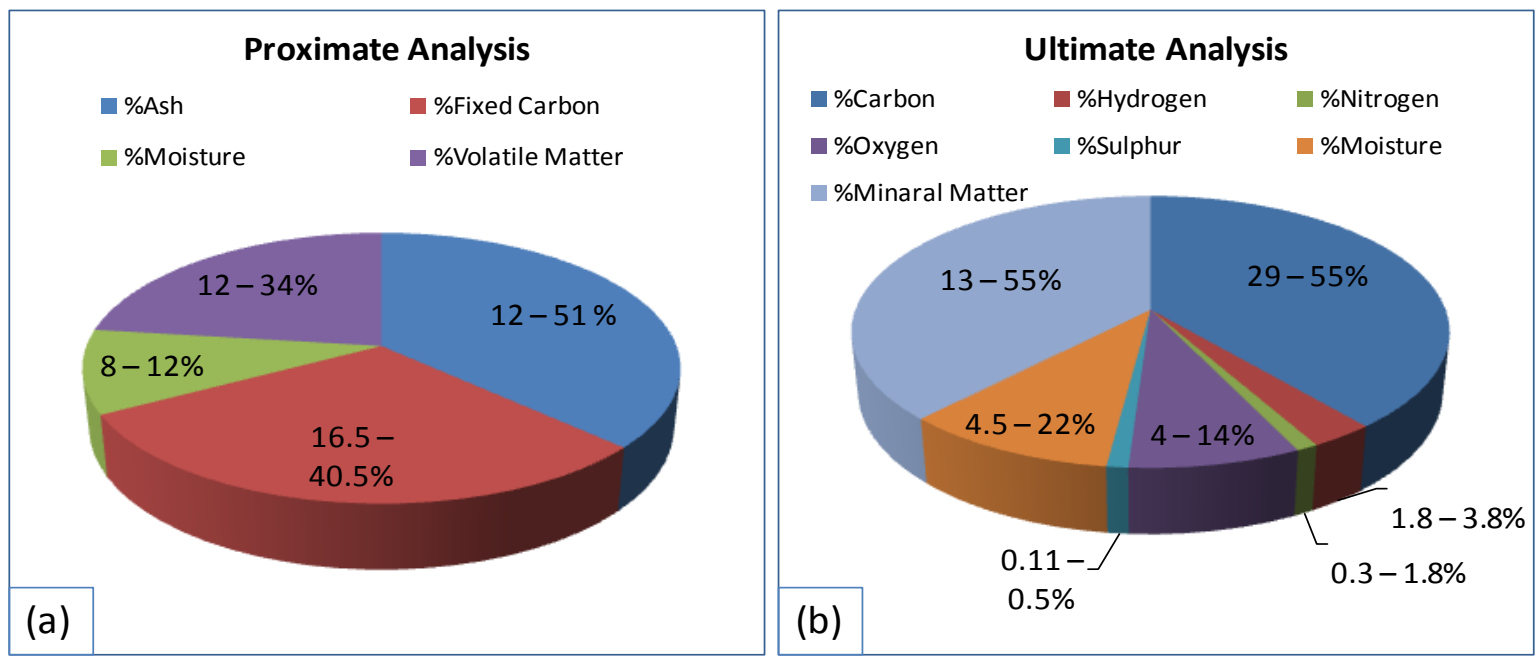

Figure 1: Range of various components for both (a) Proximate Analysis (b) Ultimate Analysis of the Indian Coal dataset used for ANN model development (source: BHEL's 150 Lab analysis data)

\section{LITERATURE REVIEW}

There are several empirical methods to compute the elemental composition (i.e. Ultimate analysis composition) based on the proximate analysis composition values. Each one of these empirical formulae may be suitable for one kind or one geographical regional based coal. Further, these have been developed based on the linearization assumptions. Aforesaid, the coal behavior is very complex in nature due to the very reason that the composition varies widely.

Artificial Neural Networks are inspired by the networks of neurons in the biological brain. Artificial neurons are a simple representation of complex biological neurons, and the network of them is called Artificial Neural Networks (ANN). There are variety of learning algorithms and architecture proposed in representing the Artificial Neural Networks and training them using the observed data respectively. Figure 2 shows a simple, generic representation ANN called Multilayered Perceptron (MLP).

In general, to address the complexity nature, researchers have explored the possibility of the using Artificial Neural Networks to model. For example, N.S. Reddy et.al. have used ANN model for modeling medium carbon steels[3], D.B. Karunakar et.al. have model effect of alloying elements on hot tearing in Aluminum casting[4]. In related to coal, S.U.Patel et.al. have used ANN for estimation of gross calorific value based on the of coal properties [5], T.Cordero et.al.[6] and Jigisha Parikh et.al.[7] have demonstrated predicting heating value from proximate analysis. Similarly, H.M. Yao et.al [8] whose model predicts percentage hydrogen in the coal based on the proximate analysis. Further, A. V. Akkaya [9] has demonstrated the possibility of predicting the High Heating Value of given coal based on the both proximate and ultimate analysis of the coal using regression method. In one more attempt, predicting the Grindability of the coal based on the petrography, proximate and ultimate analysis using multi-regression and ANN model was demonstrated by S. Chehreh Chelgani et. al [10]. Similar, work by A. K. Verma et. al. [11] to predict heat value of the coal using ANN approach. Additionally, ANN concept is being used to solve wide variety of prediction [12], estimation and controlling issues that are encountered in power-plant.
In the literature several attempts have been made to represent the proximate to ultimate composition analyses in the form of linear combinations. Each one of the approach is suitable for one geographical location of coalmines. Indian coals are typically high-ash and moisture type coals. Following formulae given by Bureau of Energy Efficiency in the guidance on their website [13] is listed in the table herein for the reference:

\section{ANN MODEL DEVELOPMENT}

With all aforesaid complexities associated to the coal properties, the relationships between the proximate and ultimate analysis compositions would be nonlinear in nature. Due to which the representations in the form of empirical equations would lead to erroneous results. As discussed in the previous sections, ANN based predictive models are powerful in terms of learning the nonlinear relationships to understand and solve, and thereby achieving ability to predict accurately. By leveraging the capability of ANN modeling technique, an ANN model is conceptualized to predict elemental composition i.e. ultimate analysis based on the available proximate analysis information. The predicted elemental data information can be used for estimating the stoichiometric air i.e. chemically balanced Oxygen needed for efficient combustion of coal along with many other controllable

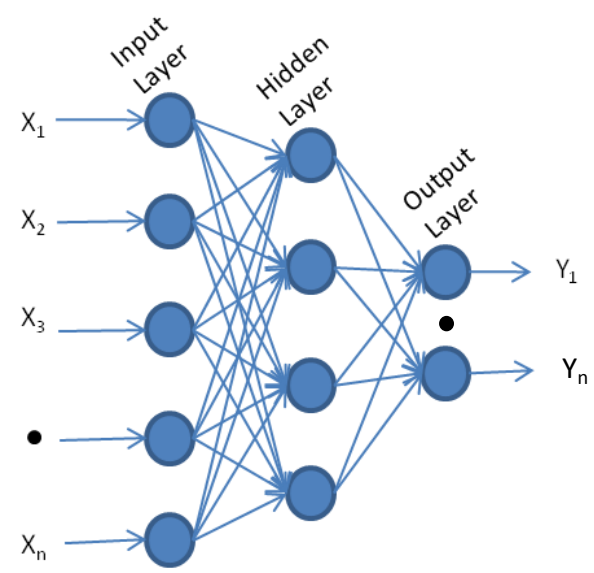

Figure 2: General ANN Architecture that represents model 


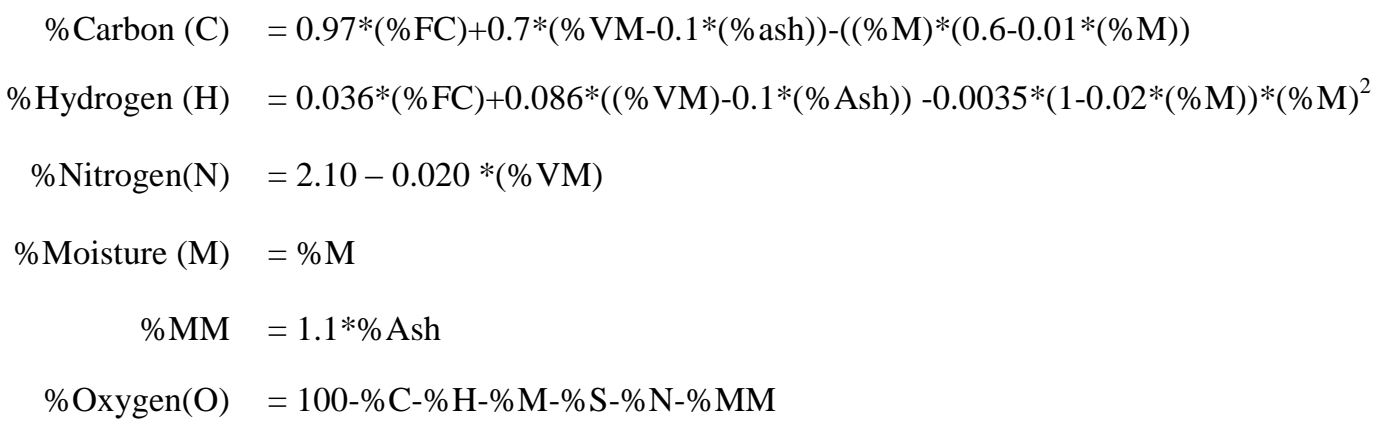

$\mathrm{M}=$ Moisture, $\mathrm{FC}=$ Fixed Carbon, $\mathrm{VM}=$ Volatile Material, $\mathrm{MM}=$ Mineral Matter

parameters.

General representation of MLP networks is shown in Figure 2. In general, MLP contains one input layer, one output layer and one or many hidden layers (Figure 2 shows single hidden layer). Depending on the number of inputs and outputs, the input neurons and output neurons are decided. There are many techniques available to identify number of Hidden Layers and neurons required to have a better learning and prediction capability of the ANN. These techniques are problem dependent, because of which fixing the number of Hidden Layers and Neurons is in general hit-and-trail method. From problem to problem number of hidden layers and neuron are identified experimentally as per the technique followed by Haykin [2007].

To start with, around $200 \mathrm{Lab}$ analyses data points were collected for the model development process. During data cleaning activity, nearly 50 lab analyses data points were found extremely different from the major data points, which were considered as outliers for the current work and discarded. Remaining 150 data points were divided into training set with 100 data points and testing set with 50 data points.

The number of the input neurons of the developed ANN model is chosen as four i.e. the gross level composition based on the proximate analysis and the output neurons are chosen as five i.e. elemental composition based on ultimate analysis. The developed model has been evolved by optimizing the number of hidden layers and hidden neurons to minimize the differences between the actual values and the predicted values in the training dataset. Using the data collected and preprocessed, the ANN model was trained and tested for its performance. Some results of the final ANN model are shown and discussed in the following section.

\section{RESULTS AND DISCUSSIONS}

ANN model performance is tested on the both training data and test data, and as well as compared with the empirical formulae to compute the elemental analysis. ANN model performance for training dataset (103 data points) is shown in Figure 3 and it has five charts that depict each one of the elemental composition. Figure 3(a) shows the estimation accuracy comparison of percentage Carbon $(\% \mathrm{C})$ of the coal based on the empirical formula and ANN prediction. With actual percentage carbon (lab data) on $\mathrm{x}$-axis, the estimations based on the empirical formula and ANN model on the y-axis are plotted. Similarly, Figure 3(b) shows percentage Hydrogen $(\% \mathrm{H})$, Figure 3 (c) shows the percentage Oxygen $(\% \mathrm{O})$ and Figure $3(\mathrm{~d})$ shows the percentage Nitrogen $(\% \mathrm{~N})$ in the coal. As shown in the Equations (1 to 6), for estimating the percentage of Sulphur there is no direct empirical formula.
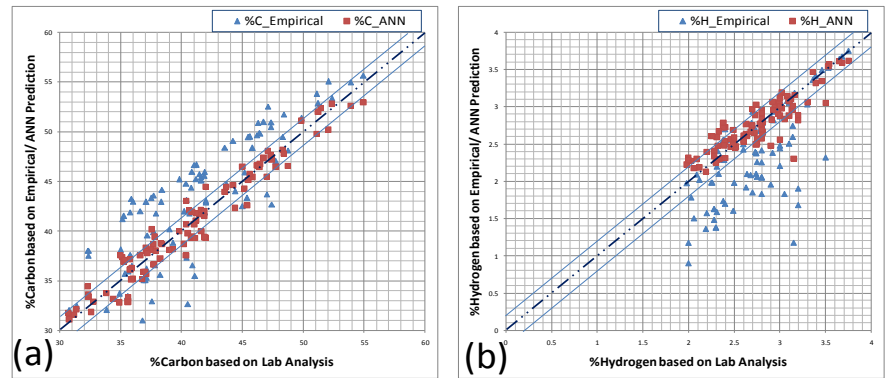

(b) \%Hydrogen based on Lab Analysis

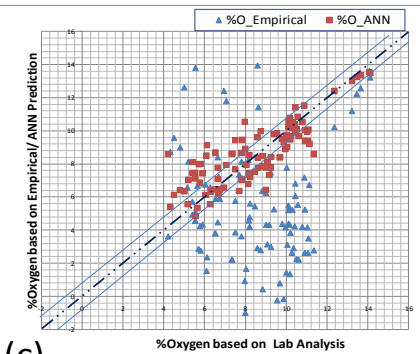

(c)
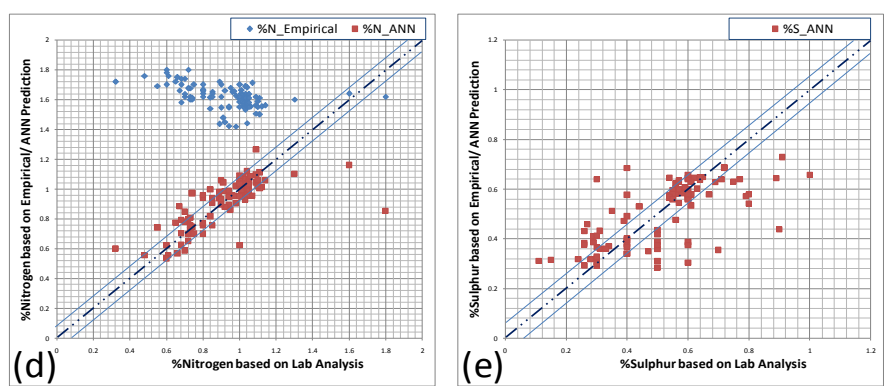

Figure 3: Chart depicting the results of estimations and predictions on Training dataset by both empirical formulae and ANN model overlaid together to have better comparison (a) \%Carbon, (b) \%Hydrogen, (c) \%Oxygen and (d) \% Nitrogen; (e) ANN prediction of \% Sulphur (no empirical formula available) 

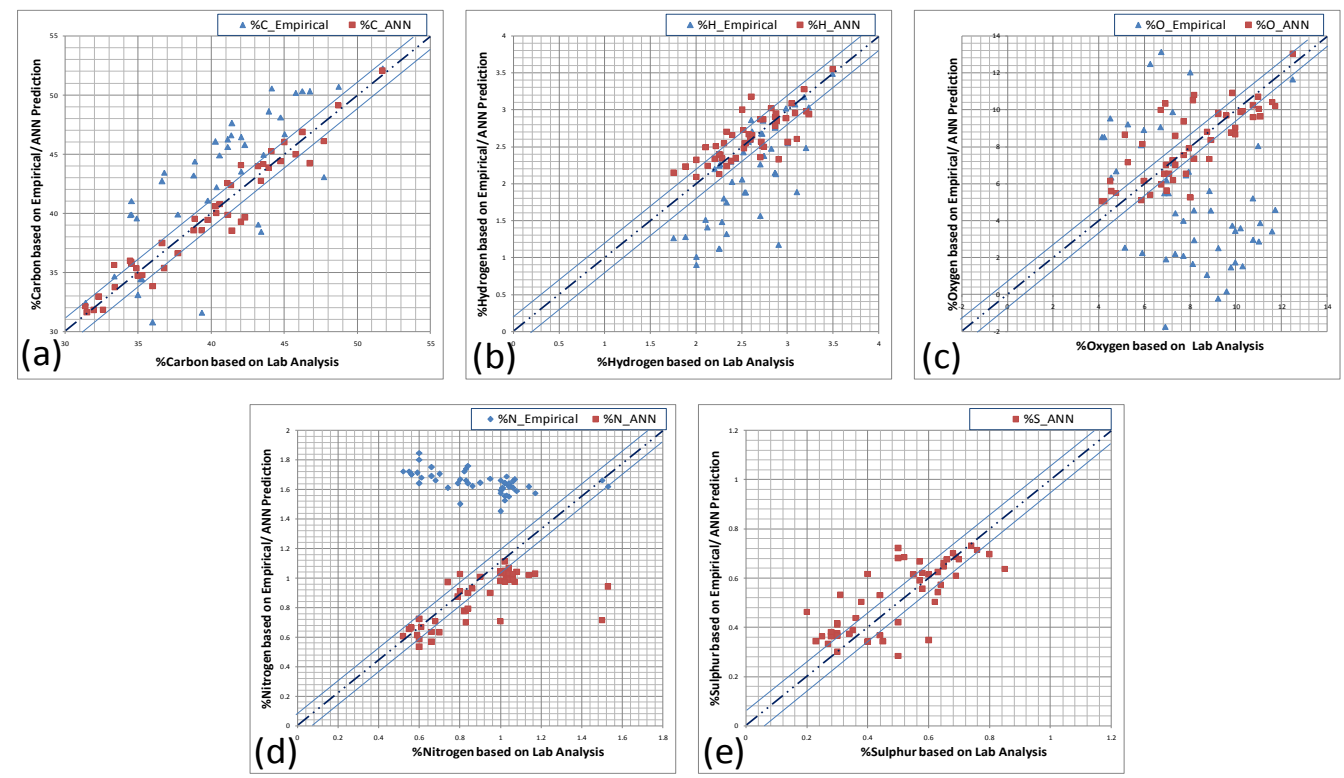

Figure 4: Chart depicting the results of estimations and predictions on Testing dataset by both empirical formulae and ANN model overlaid together to have better comparison (a) \% Carbon, (b) \%Hydrogen, (c) \%Oxygen and (d) \% Nitrogen; (e) ANN prediction of \% Sulphur (no empirical formula available)

Therefore, Figure 3 (e) shows the ANN model predictions in comparison with actual analysis values of percentage Sulphur $(\%$ S). Similarly, Figure 4 show the ANN performance on test dataset (50 data points) and all five chart of the elemental composition are displayed in the sequence as that of Figure 3. Predictions falling close to the 45 degree line on all the Figure 3(a) to (e) and Figure 4 (a) to (e) indicate accurate prediction, i.e. with minimum error in the prediction. All the deviations from that line indicate error in the predictions; which could be positive or negative error in the estimation. Overall, it can be clearly noticed that the ANN model has predictions less deviated from the actual analysis values compared with the empirical formula based predictions. Triangle shaped points in the figures indicate the prediction by empirical formulae and rectangle shaped points indicate prediction by ANN model. For the performance check two parallel lines as band on both sides of the 45 degree line are drawn to indicate the best performance band. It has been observed that more than $80 \%$ of the data points of ANN predictions are within the $\pm 20 \%$ of error. For more clarity Table 1 is provided showing the percentage of data points falling within the $\pm 20 \%$ error range. It also, clear that majority of empirical formulae based predictions are much outside the $\pm 20 \%$ error range. The performance of the ANN model further can be improved by adding more training data points of carefully performed coal analysis.

\section{CONCLUSION}

Under this work, an ANN model has been developed for predicting ultimate analysis of the coal with proximate analysis as input. The developed ANN model gave a better performance in predicting the values of the Ultimate analysis composition when compared with empirical formulae based estimation of the same. The model can be used by boiler designer at design stage to estimate the combustion air requirement when there is only proximate analysis of the linked-coal is available. Also, the boiler operator can use this model to achieve better operational controls for improved performance. At thermal power plants, wherever online proximate analysis information is available, the model output can be used to arrive at ultimate analysis and thereby improving combustion efficiency by controlling excess air and other related control parameters.

\section{ACKNOWLEDGMENTS}

Authors would like to express their gratitude to BHEL management for approving to publish the research work conducted.

\section{REFERENCES}

[1] ASTM, Standard Practice for Proximate Analysis of Coal and Coke, (2007), D 3172 - 07a.

[2] M.C. Mayoral, M.T. Izquierdo, J.M. Andrés, B. Rubio, Different approaches to proximate analysis by thermogravimetry analysis, Thermochimica Acta, Volume 370, Issues 1-2, 4 April 2001, Pages 91-97

[3] N.S.Reddy, J.Krishnaiah, Seong-Gu Hong, Jae Sang Lee, Modeling medium carbon steels by using artificial neural networks, Materials Science and Engineering A, Volume 508, Issues 1-2, 20 May 2009, Pages 93-105. (Online copy: http://dx.doi.org/10.1016/j.msea.2008.12.022)

[4] D. Benny Karunakar, J.Krishnaiah, S.Patra and G.L. Datta, Effects of Grain Refinement and Alloying Elements on Hot Tearing in Aluminum Casting, International Journal of Production and Quality Engineering, Vol. 1, No. 1, Jan-June 2010, Pages: 13-20

[5] Shagufta U. Patel, B. Jeevan Kumar, Yogesh P. Badhe, B.K. Sharma, Sujan Saha, Subhasish Biswas, Asim Chaudhury, Sanjeev S. Tambe, Bhaskar D. Kulkarni, Estimation of gross calorific value of coals using artificial neural networks, Fuel, Volume 86, Issue 3, February 2007, Pages 334-344, ISSN 0016-2361, 10.1016/j.fuel.2006.07.036.

(http://www.sciencedirect.com/science/article/pii/S00162 36106002961)

[6] T Cordero, F Marquez, J Rodriguez-Mirasol, J.J Rodriguez, Predicting heating values of lignocellulosics 
and carbonaceous materials from proximate analysis, Fuel, Volume 80, Issue 11, September 2001, Pages 1567-1571, DOI: 10.1016/S0016-2361(01)00034-5

[7] Jigisha Parikh, S.A. Channiwala, G.K. Ghosal, A correlation for calculating HHV from proximate analysis of solid fuels, Fuel, Volume 84, Issue 5, March 2005, Pages:487-494, DOI: 10.1016/j.fuel.2004.10.010 (http://www.sciencedirect.com/science/article/pii/S00162 36104003072)

[8] H.M. Yao, H.B. Vuthaluru, M.O. Tadé, D. Djukanovic, Artificial neural network-based prediction of hydrogen content of coal in power station boilers, Fuel 84 (2005) $1535-1542$

[9] Ali Volkan Akkaya, Proximate analysis based multiple regression models for higher heating value estimation of low rank coals, Fuel Processing Technology, (2009), 165 $-170$
[10] S. Chehreh Chelgani, James C. Hower, E. Jorjani,, Sh. Mesroghli, A.H. Bagherieh, Prediction of coal grindability based on petrography, proximate and ultimate analysis using multiple regression and artificial neural network models, FUEL PROCESSING TECHNOLOGY 89 (2008) $13-20$

[11] A. K. Verma, T. N. Singh and M. Monjezi, Intelligent prediction of heating value of coal, Iranian Journal of Earth Sciences 2 (2010), 101-109

[12] Simon Haykin, Neural Networks: A Comprehensive Foundation (3rd Edition), Prentice-Hall, 2007, ISBN:0131471392

[13] Bureau of Energy Efficiency, Fuels and CombustionChapter: http://www.em-ea.org/Guide\%20Books/book$2 / 2.1 \% 20$ Fuels $\% 20$ and\%20combustion.pdf 